\title{
Reliability Analysis of Light Emitting Diode Technologies for Cabin Lighting in Manned Space Flight Applications
}

\author{
Todd H. Treichel ${ }^{1}$ \\ Orbital Technologies Corporation, Madison, Wisconsin 53717
}

\begin{abstract}
Light emitting diode (LED) technology is used by the commercial markets to replace traditional fluorescent and incandescent lighting technologies for consumers to take advantage of energy savings. Advantages of transitioning to LED technologies in spacecraft are reduced mass, reduced occupied volume, reduced power, improved color control, longer operating life, and lower cost associated with power consumption and disposal. Components and subsystems that make up the Environmental Control and Life Support System (ECLSS) require continued performance and operation. A system failure cannot be tolerated for it is extremely difficult to repair and expensive to replace. Therefore, the vehicular LED module must function when launched and continue to function throughout the respective product life-cycle (days, month, or years depending on mission). This analysis evaluates three different LED package types for reliability performance using Electrical, Electronic, and Electro-mechanicals (EEE) parts testing techniques. The goal of the study was to investigate the suitability of commercial off the shelf (COTS) LED technologies in accordance with a probable EEE parts management plan.
\end{abstract}

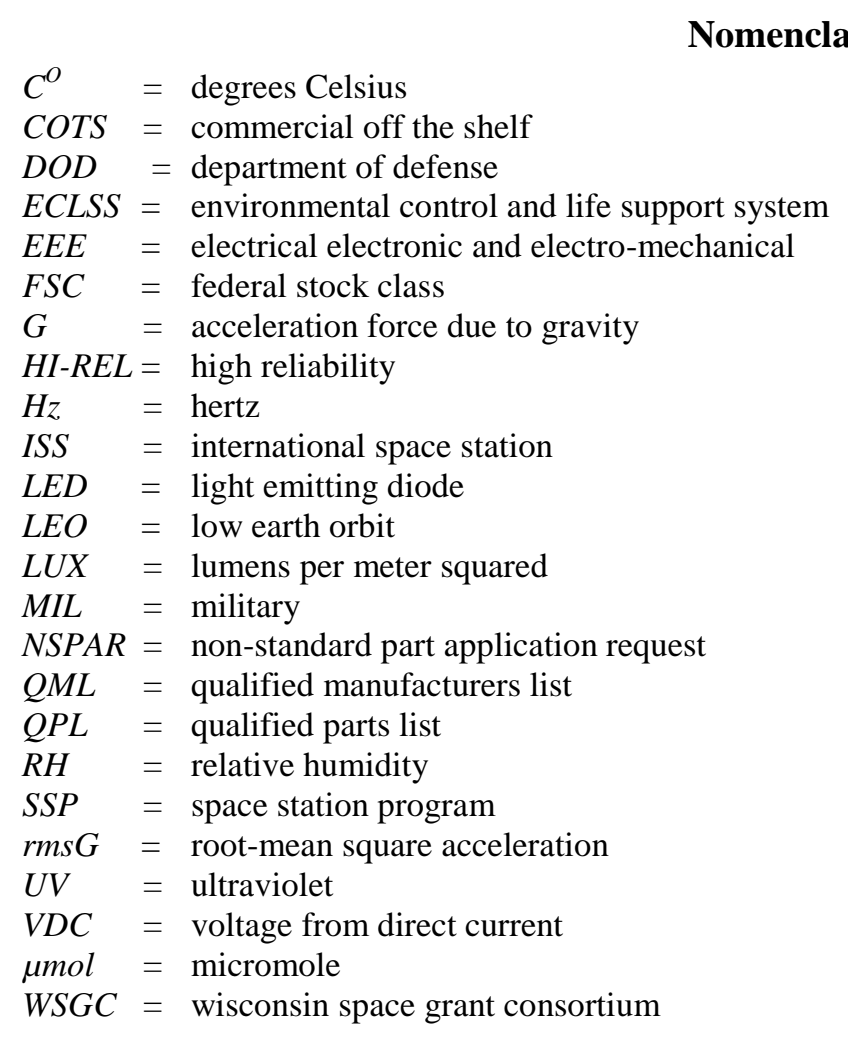

\footnotetext{
${ }^{1}$ Systems Engineer, Space Center, 1212 Fourier Drive, Madison, Wisconsin, USA
} 


\section{Introduction}

$\triangle$ EROSPACE and military applications are those where continued performance and operation -on-demand is critical, equipment downtime cannot be tolerated, end-use environments may be uncommonly harsh, and the equipment must function when required (Hersman \& Fowler, 2009). Technology in the electronics industry changes rapidly and engineers within the aerospace industry do not always have access to new technology due to high-reliability (HI-REL) requirements established in DOD and NASA procurement standards. Engineers designing for commercial products can experiment with new

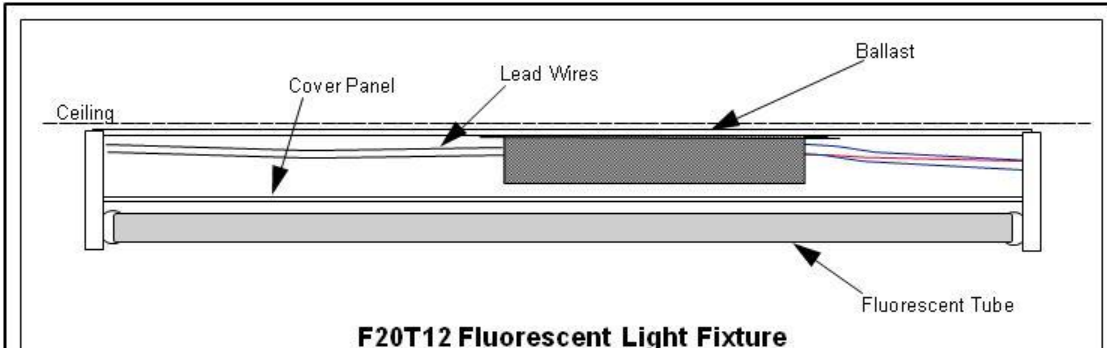

F20T12 Fluorescent Light Fixture

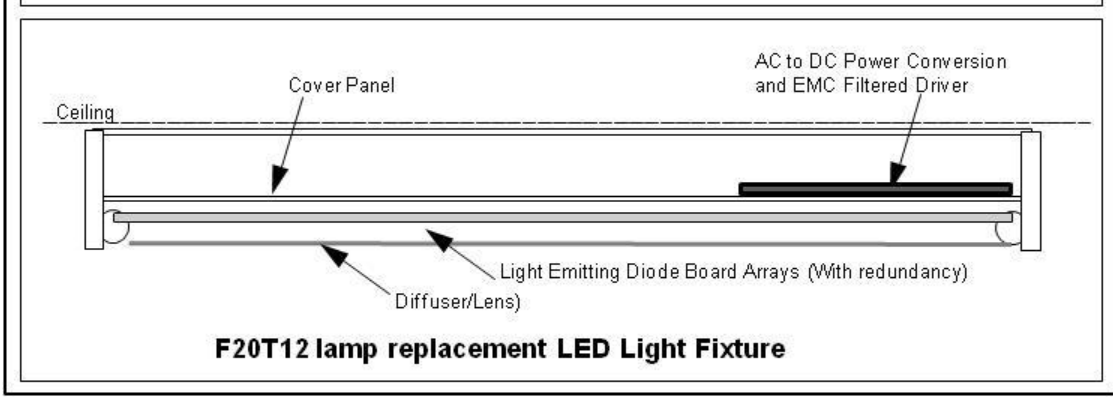

Figure 1. Schematic of fluorescent light replaced with LED technology.

technologies with little or no restrictions in design component selection. Military and aerospace engineers are required to manage product designs by making component selections from DOD and NASA approved part lists, where specific components have been tested extensively and heritage field data is cataloged for quality and reliability performance. New electronic technologies are being developed and by the time these technologies become available on a DOD quality parts list (QPL) or quality manufacturer's list (QML), the technology may have been replaced or improved in the commercial markets, thus making it difficult for aerospace engineers to select state-of-the-art design components without extensive testing. This analysis investigates the suitability of selecting white LED components from three leading manufacturers currently supplying commercial markets.

\section{Light Emitting Diodes}

Light emitting diodes are semiconductors that convert electrical energy into light energy. The color of the emitted light is designed into a specific semiconductor material composition for each component where individual LEDs may be selected for mixing of colors to obtain a desired color of light output. Light emitting diodes are classified into ultraviolet, visible, and infrared wavelengths depending on the technological application (Lenk \& Lenk, 2011).

Development of high-power LED technology involves challenges for design engineers in that LED lighting devices are subject to high temperatures that must be properly managed. Increased junction temperatures of the LED chip, causes stress on associated material and may cause earlier than expected light output degradation which will lead to an operational failure. Lenk and Lenk (2011) described two primary methods for producing high intensity white-light using LED technology: (1) one is to use individual LEDs that emit the three primary colors of red, green, and blue, and mix the three colors to produce white light, and (2) is to use a phosphor material to convert monochromatic light from a blue or ultraviolet (UV) LED to broad-spectrum white light. The latter of these two primary methods are applicable to the investigation

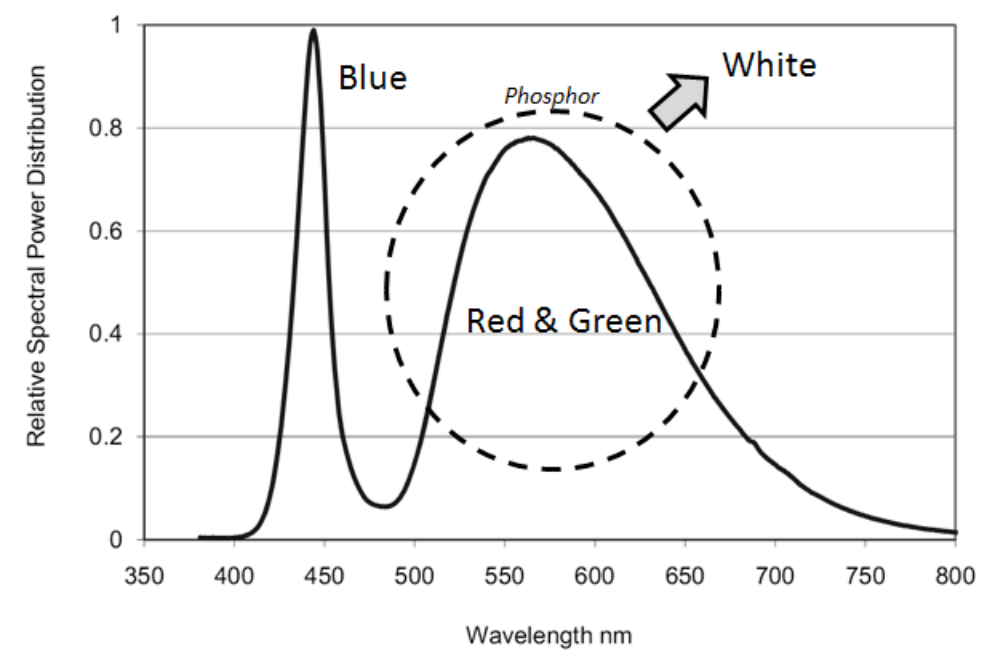

Figure 2. Broad-spectrum white light color distribution model. 
discussed herein.

Light emitting diode technology can provide the military and space community with advantages when compared to the current use of incandescent light sources including: (a.) lower power consumption, (b) improved reliability by use of redundancy, (c) improved ruggedness for harsh environments, (d) lighter weight, (e) smaller size, (f) improved control over color and brightness and, (g) faster switching. Figure 1 illustrates the differences between a fluorescent light fixture, commonly used in military applications such as US Navy vessels, and the smaller, and lighter weight LED technology which may be retrofitted into an existing fluorescent light fixture.

\section{Purpose of Investigation}

Advantages, similar to those previously mentioned, have been noted by Brainard, Coyle, Ayers, Kemp, Warfied, Maida, Bowen, Bernecker, Lockley, \& Hanifin (2012). The International Space Station (ISS) contains fluorescent light technologies for illuminating the astronauts' research and living environments NASA engineers have built a case for replacing fluorescent lighting with LED technology due to advantages such as: (a) lower heat generation, (b) lower power consumption, (c) less weight, (d) greater resistance to damage, (e) less toxic material, (f) elimination of fluorescent tube disposal, and (g) improved reliability. The fluorescent lamp technology on ISS is aging and there is an acceleration of failures which require replacement, due to a deficiency of NASA flight qualified fluorescent units, LED technologies provides an opportunity for retrofitting the ISS with lighting that has improved efficiency, no mercury, and improved reliability (Brainard et al., 2012). Little is known about which commercially available LED components are the most robust in accordance with aerospace requirements. This information will prove useful for future commercial space applications which may include both vehicles and habitable structures.

\section{Flexible Path Space Missions}

In September of 2009 the 156 page Augustine report was published, by the US Government Printing Office, documenting a new space exploration concept called flexible path. Flexible path is a concept that involves human space flight vehicles and missions travelling beyond low-Earth-orbit (LEO) and unlike past space missions managed by NASA, development of space vehicles, rocket engines, and instrumentation will be the responsibility of commercial companies with NASA oversight (Szajnfarber, Coles, Sondecker, Wicht, \& Weigel, 2011). Commercial aerospace companies are to be utilized, in lieu of NASA resources, in an effort to save costs and add flexibility with choices in technology and mission objectives. Flexible path missions require commercial crew launches to LEO, followed by further technology developments for exploration to destinations farther away from Earth, such as asteroids, the Lunar surface, and Mars (Szajnfarber et. al, 2011).

Literature contained in the Augustine report provides a baseline for this analysis. Research and analysis of LED technology is justified with the intent of meeting human space flight goals by conducting development and mission operations with improved efficiency and lower cost. Flexible path missions will involve risk, management of lifecycle cost, and development of reliable equipment capable of reliable on-demand-operation over the course of the entire mission.

\section{Aerospace Technology Readiness}

Hersman and Fowler (2009) emphasized that electronics on spacecraft must be able to withstand the space environment to assure mission success and meet design criteria that is more robust than equivalent commercial products available for terrestrial applications. Engineers working in the aerospace and military industries, order relatively low quantities of space qualified electrical components, often at irregular intervals, with long lead times, and high dollar costs for specialized manufacturing. Light emitting diodes remain an unproven technology in the aerospace industry, where commercial manufacturers are the only source for procurement and require research for space flight suitability. Engineers are required to reference NASA or DOD approved parts lists and select parts based on flight heritage and proven reliability. Approved components are termed high reliability (HI-REL) and are listed by Federal Stock Class (FSC) numbers on lists called quality parts list (QPL) and quality manufacturer's list (QML), managed by the US Defense Logistics Agency (DLA). No FSC number exists for LED component technologies. These parts lists are updated periodically and inclusion of new technology is slow due to lack of historical flight or reliability data.

Advancements in technologies are made in the commercial sector where state-of-the-art component technologies are not accessible to aerospace engineers when designing space flight hardware. Selection of commercial parts to be considered grade level three (see Table 1) because proof-of-concept and reliability test data are not available for reference, thus rendering these new technologies unavailable for immediate use. The only 
alternative is to select a commercial off the shelf (COTS) component and perform electrical, electronic, and electromechanical (EEE) qualification testing to provide evidence that selected components are capable of meeting specified mission requirements.

Selecting grade level three parts is the least desirable option for engineer component selection and a literature review of NASA parts management documents yielded that there are no specified test paths for qualifying LED technology for space flight. Grade level three parts are classified as the highest risk selection. Table 1 illustrates criteria of grade level classifications assigned to component reliability. Parts not EEE listed are to be evaluated for space flight heritage, similarity, and existing test data for the purpose of classifying parts to desired grade level.

\section{Table 1. Key requirements for project initiation and management}

\begin{tabular}{cl}
\hline Grade Level & \multicolumn{1}{c}{ Mission Selection Criteria } \\
\hline 1 & $\begin{array}{l}\text { Components selected for mission application requiring the highest reliability and lowest } \\
\text { level of risk. Typical mission durations are five years or longer. }\end{array}$ \\
2 & $\begin{array}{l}\text { Components selected for low to moderate risk. Selection is to be balanced by cost } \\
\text { constrains and mission objectives. Reliability and performance data specific to desired } \\
\text { mission may not be available and some testing may be required. Typical mission durations } \\
\text { are one to five years. }\end{array}$ \\
& $\begin{array}{l}\text { Components selected for mission represent inherently high risk unknown risk due to lack of } \\
\text { formalized reliability assessment, screening, and qualification. Available data does not } \\
\text { address flight history and construction materials, manufacturing, and design processes are } \\
\text { in continuous change, which is unreliable due to lack of consistency and process } \\
\text { repeatability. Level three components are the least desirable for use where typical mission } \\
\text { durations are less than one to two years. }\end{array}$ \\
\hline
\end{tabular}

\section{EEE Test Plan}

Components used for flight design and construction are evaluated for HI-REL heritage and suitability. Documents such as SSP 30312 Electrical, Electronic and Electromechanical (EEE) and Mechanical Parts Management and Implementation Plan for Space Station and NASA-EEE-INST-002 Instructions for EEE Parts Selection, Screening, Qualification, and Derating are typically referenced for managing qualification and upscreening techniques for evaluation of COTS parts usage in flight system designs.

When approved within the non-standard part application request (NSPAR) document, screening tests may be employed with the intent to remove nonconforming parts (parts with random defects that are likely to result in early failures, known as infant mortality) from an otherwise acceptable lot and thus increase confidence in the reliability. A technology review board holds authority over NSPAR approval where certain part requirements may not be sufficient, depending on the specific device construction, mission life and reliability goals of the project. For the purpose of this analysis no NSPAR documents were involved and for the promotion of science and not LED companies, product brand names have been omitted from the three selected LED manufacturers selected for investigation. Figure 3 illustrates graphical representations for the three different LED package types, purchased from industry leading manufacturers, for the purpose of this EEE evaluation.

Environmental tests are applied to accelerate the aging rate of a product by elevating and/or cycling the product temperature. The process may be amplified by introducing other environmental factors such as vibration and shock etc. Regardless of the technique employed, the purpose of environmental testing is twofold:

1) To weed out the early life failures. The intent is to detect non-conformances before the product is shipped to the customer. By doing this, the customer should experience a low failure rate characterized by the useful product life.

2) To provide a feedback mechanism, whereby test failures are analyzed and appropriate design and process changes are implemented prior to continuous manufacturing of a released design. 
SSP 30312, NASA-EEE-INST-002, and similar EEE parts management plans typically reference a FSC number for an appropriate qualification and screening test path. Federal stock class number 5961 references diodes, where this researcher determined that an LED package is not applicable to the composition of a two-terminal part designed to direct circuit current flow in one direction. An LED provides a semiconductor based light source, a review of Figure 3 reveals design construction containing a miniaturized electronic circuit bonded to a substrate containing a cavity, semiconductor material layer, wire bonds, and epoxy encapsulation. For this reason this researcher chose FSC 5962 for hybrid microcircuits as the most applicable FSC reference for EEE testing. Lenk and Lenk (2011) supported this view by stressing the fact that some LED packages contain bond wires that connect the dies to leads for the purpose of putting current through the circuit where a single device contains multiple bond wires used in parallel to accommodate relatively high currents.

Referencing MIL-PRF-38534 General Specification for Hybrid Microcircuits Table 2 was constructed and summarizes the implemented test plan. Environmental tests were selected by choosing those conditions deemed suitable for qualification and for the purpose of investigation potential failure modes. For example, MIL-PRF-38534 stated that constant acceleration or mechanical shock could be administered and in this analysis both tests were performed.

Table 2. Test plan.

\begin{tabular}{|c|c|c|c|c|}
\hline \multirow[t]{2}{*}{ Subgroup } & \multirow[t]{2}{*}{ Test } & \multicolumn{2}{|r|}{ MIL-STD-883 } & \multirow{2}{*}{$\begin{array}{c}\text { Quantity } \\
\text { (Accept } \\
\text { Number) }\end{array}$} \\
\hline & & Method & Condition & \\
\hline \multirow{10}{*}{1} & Electrical Verification & - & - & \multirow{10}{*}{$5(0)$} \\
\hline & External Visual Inspection & 2009 & Examined at $1.5 \mathrm{X}$ to $10 \mathrm{X}$ magnification. & \\
\hline & Temperature Cycling & 1010 & Cond. C: 20 Cycles & \\
\hline & Constant Acceleration & 2001 & Cond. A: 5000 g's, Y axis & \\
\hline & Mechanical Shock & 2002 & Cond. B: 1500 g's & \\
\hline & Random Vibration & 2026 & Cond. F: 20.0 GRMS & \\
\hline & Sinusoidal Vibration & 2005 & Cond. A: 20 g's & \\
\hline & Moisture Resistance & 1004 & 24 Hours & \\
\hline & Barometric Pressure & 1001 & Cond. G: $2.4 \times 10^{-6} \mathrm{~mm}$ Mercury & \\
\hline & End Point Electrical & - & - & \\
\hline \multirow{3}{*}{2} & Electrical Verification & - & - & \multirow{3}{*}{$22(0)$} \\
\hline & Steady State Life Test & 1005 & Cond. B: 1000 hours, $125^{\circ} \mathrm{C}$ & \\
\hline & End Point Electrical & - & - & \\
\hline
\end{tabular}
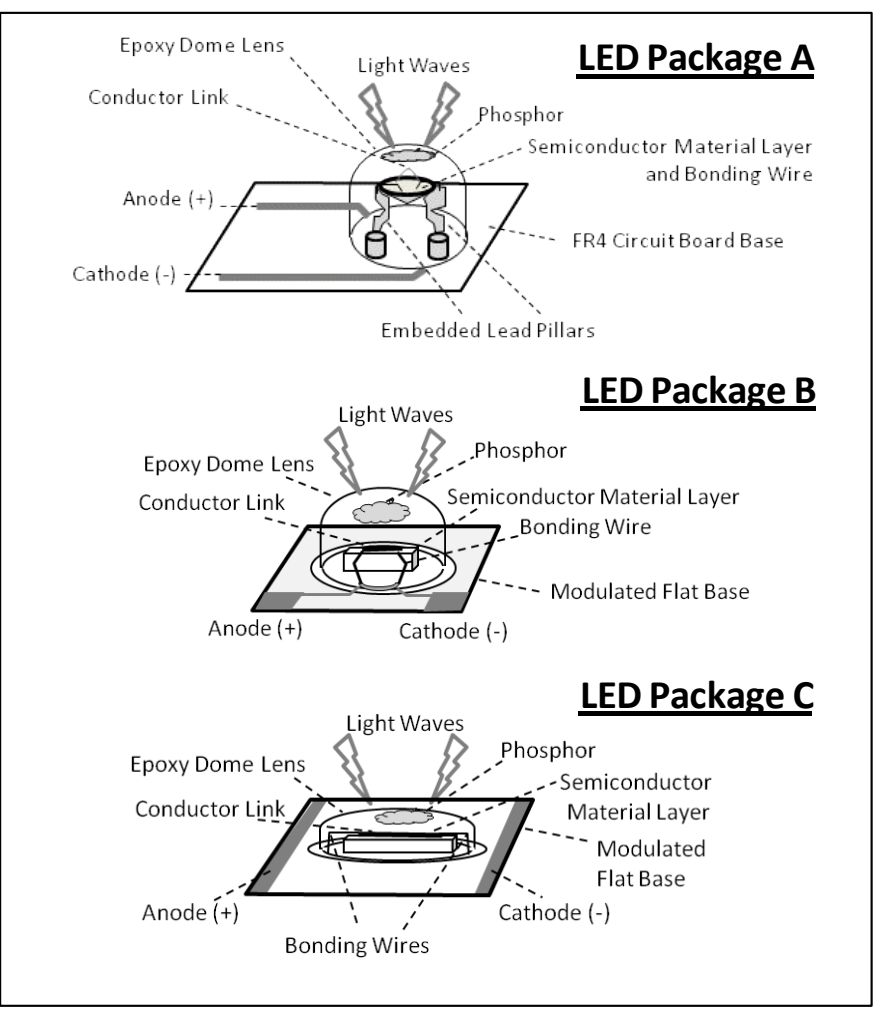

Figure 3. Representation of different LED packages under test. 


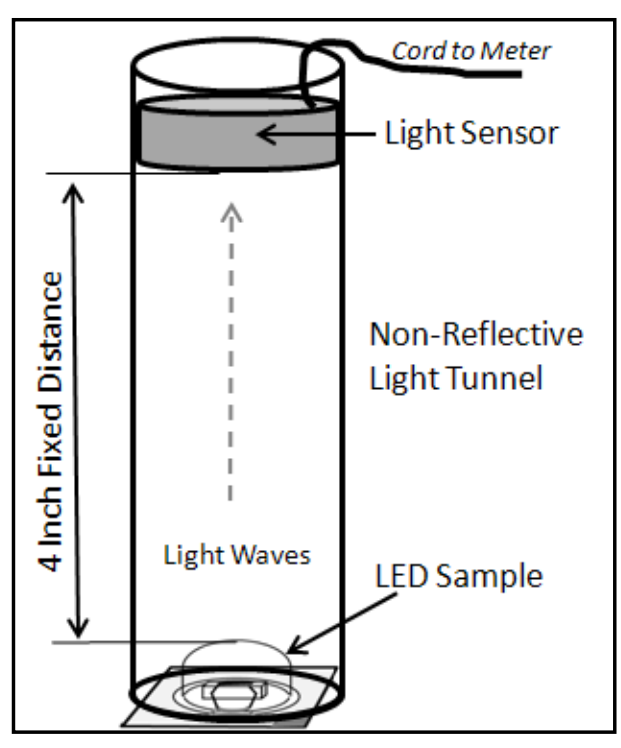

Figure 4. Light output measurement configuration.

tested in search of device failures and a visual examination was conducted in search of mechanical damage. No failures were found.

\section{B. Constant Acceleration}

The purpose of constant acceleration testing is to determine the effects on the types of structural and mechanical weaknesses not necessarily detected in vibration tests. It may be used as a high stress test to determine the mechanical limits of the package, internal metallization, and lead system, die or substrate attachment, wire bond attachment, and other elements of the device.

Samples were placed into a centrifuge and subjected to constant acceleration per, MIL-STD 883, method 2001, $\mathrm{Y}_{1}$ axis only, for one (1) minute at fivethousand (5000) g's. Figure 11 provides a schematic outlining the positioning of each LED device in the $\mathrm{Y}_{1}$ direction. Upon completion the LED devices were electrically tested in search of device failures and a visual examination was conducted in search of mechanical damage. No failures were found.

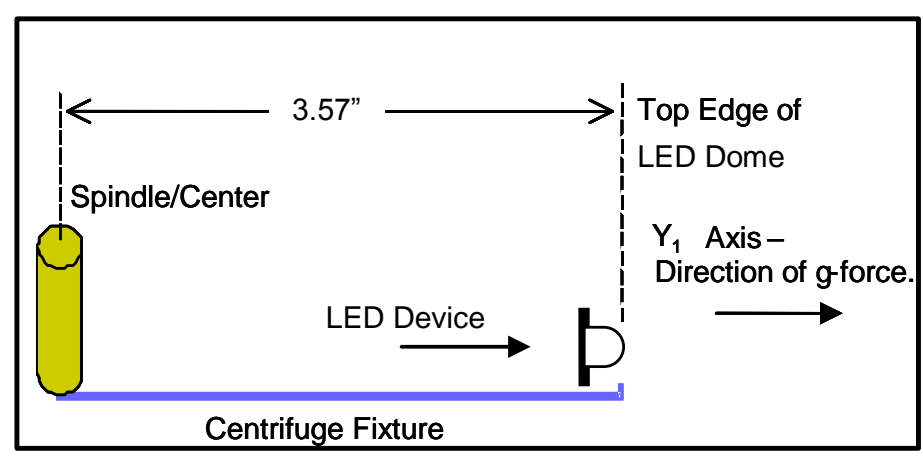

Figure 6. Constant acceleration centrifuge configuration.

\section{Mechanical Shock}

The purpose of mechanical shock is to determine the suitability of the devices for use in electronic equipment which may be subjected to moderately severe shocks as a result of suddenly applied forces or abrupt changes in motion produced by rough handling, transportation, or field operation. Shocks of this type may disturb operating characteristics or cause damage similar to that resulting from excessive vibration.

Samples were mounted in a fixture and 


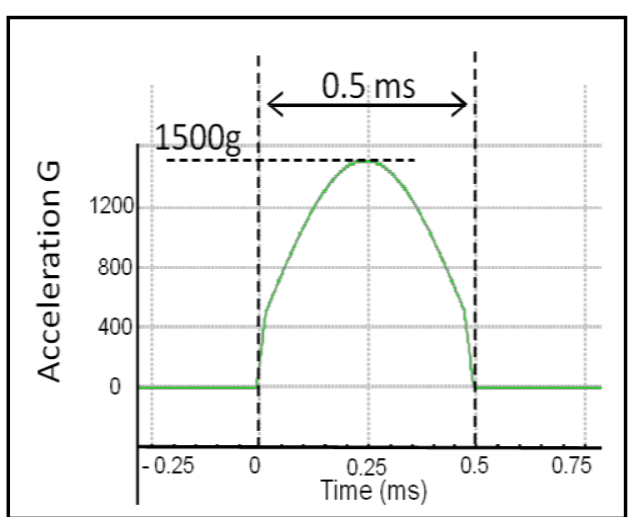

Figure 7. Shock half sine pulse.

placed onto a Mechanical Shock machine and shock tested to MIL-STD-883, method 2002, condition A. Figure 7 illustrates the one-thousand five-hundred $(1,500) \mathrm{G}$ shock profile executed on each of six (6) mutually perpendicular axes. Positive and negative pulses were tested by applying five (5) shocks for each axes respectively. Upon completion the LED devices were electrically tested in search of device failures and a visual examination was conducted in search of mechanical damage. No failures were found.

\section{Random Vibration}

The purpose of random vibration testing is to determine the ability of the device to withstand the dynamic stress exerted by random vibration applied between upper and lower frequency limits $(20-2000$ hertz $(\mathrm{Hz})$ using the power spectral density) to simulate the vibration experienced in various field environments.

Random vibration is characteristic of modern field environments produced by manned-launch vehicles, unmanned cargo vehicles, missiles, high-thrust jets and rocket engines.

The LED samples were placed on a vibration shaker table and tested in accordance with MILSTD-883, method 2026, condition F. Figure 8 illustrates the 20.0 GRMS random profile that was performed on each of three (3) mutually perpendicular axes for fifteen (15) minutes each. Upon completion LED devices were electrically tested in search of device failures and a visual examination was conducted in search of mechanical damage. No failures were found.

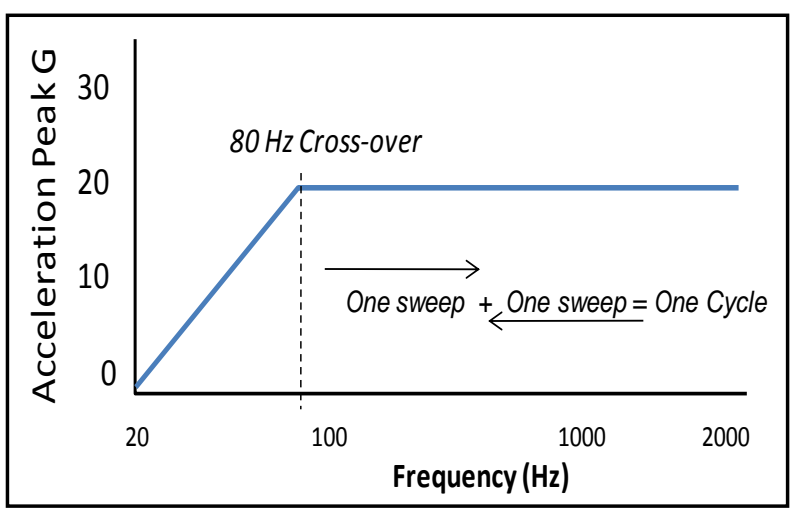

Figure 9. Sinusoidal vibration profile. executed on each of three (3) mutually perpendicular axes. A total of six (6) cycles were administered at twenty (20) G's. Upon completion LED devices were electrically tested in search of device failures and a visual examination was conducted in search of mechanical damage. No failures were found.

\section{F. Moisture Resistance}

A moisture resistance test is performed for the purpose of evaluating, in an accelerated manner, the resistance of component parts and constituent materials to the deteriorative effects of high-humidity and heat conditions. Most material degradation results directly or indirectly from absorption of moisture vapor and films by vulnerable insulating materials, and from surface wetting of metals and insulation. These phenomena produce many types of deterioration, including corrosion of metals, constituents of materials, and detrimental changes in electrical 
properties. This test differs from the steady-state humidity test and derives its added effectiveness in its use of temperature cycling, which provides alternate periods of condensation and drying essential to the development of the corrosion processes and produces a breathing action of moisture into partially sealed enclosures. Increased effectiveness is also obtained by use of a higher temperature, which intensifies the effects of humidity. This test included a low-temperature sub-cycle that served as an accelerant to reveal otherwise indiscernible evidences of deterioration caused by freezing moisture, which tends to widen cracks and fissures.

The LED samples were placed into a humidity test chamber and subjected to twenty-four (24) hours of humidity cycling in accordance with the profile illustrated in Table 3. Upon completion the LED device were electrically tested in search of device failures and a visual examination was conducted in search of mechanical damage. No failures were found.

Table 3. Humidity cycling test profile per MIL-STD-883, test method 1004.

\begin{tabular}{|c|c|c|c|}
\hline STEP & $\begin{array}{c}\text { TEMPERATURE } \\
\text { (In deg C) }\end{array}$ & $\begin{array}{c}\text { HUMIDTIY } \\
\text { (In \% RH) }\end{array}$ & $\begin{array}{c}\text { TIME } \\
\text { (In hours) }\end{array}$ \\
\hline 1 & 125 & NA & 24 \\
\hline 2 & 25 to 65 & 0.0 to 95 & 3.5 \\
\hline 3 & 65 & 95 & 2.5 \\
\hline 4 & 65 to 25 & 95 & 2.5 \\
\hline 5 & 25 to 65 & 95 & 3.0 \\
\hline 6 & 65 & 95 & 2.5 \\
\hline 7 & 65 to 25 & 95 & 1 \\
\hline 8 & 25 & 95 to 0.0 & 3 \\
\hline 9 & 25 to -10 & 0.0 & 1 \\
\hline 10 & -10 & 0.0 to 95 & 2 \\
\hline 11 & -10 to 25 & 95 & \\
\hline 12 & 25 & & \\
\hline
\end{tabular}

\section{G. Barometric Pressure Altitude Test}

The purpose of vacuum testing is to determine the suitability of component parts and subassemblies for flightworthiness in avionics applications or exposure to space vacuum. Differential pressure is used to simulate exposure to varying altitudes and seek information about adverse effects and unwanted failure modes. Devices were place into a vacuum chamber (see Figure 10) and tested in accordance with MIL-STD883, method 1001, condition G. The LED samples were powered in compliance with operating specifications and subjected to an atmospheric pressure of (760 Torr). Within ten (10) minutes the test environment transitioned to a vacuum environment of $\leq 10^{-6}$ Torr for a total duration of one (1) hour. The LED device continued to successfully operate during the entire test duration. Upon removal from the vacuum vessel, a visual examination was conducted in search of mechanical damage. No failures were found.

\section{H. Steady State Life Test}

The purpose of powered life testing is to screen for and eliminate marginal devices, those with inherent defects or defects resulting from manufacturing weaknesses, which cause time and stress dependent failures. The theory behind this activity is to verify performance capability and eliminate infant mortality

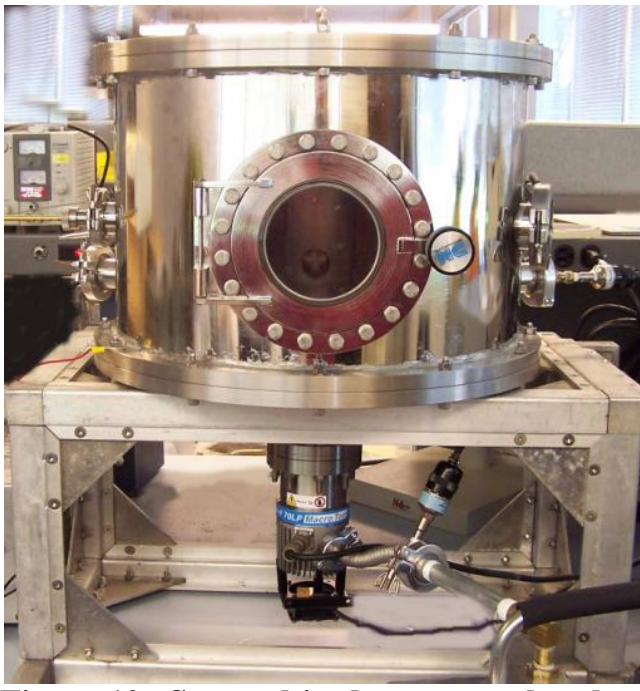

Figure 10. Space altitude vacuum chamber. (early life cycle failures) with the intent of stressing electronics in an accelerated manner, which will reveal time and stress dependent failure modes. Steady state life testing is a test technique considered to be longer than a typical burn-in with the intent of seeking information about qualification 
and acceptance for space flight usage. Samples were placed into an environmental test chamber and exposed to $+125^{\mathrm{O}} \mathrm{C}$ for 1000 hours of powered operation in accordance with MIL-STD-883, method 1005, Condition B (forward bias). The $+125^{\circ} \mathrm{C}$ test environment is also the same temperature required for Class $S$ (space) rated components when qualifying to a QML. It should be noted that 22 LED samples were selected for powered life testing, separate from those exposed to the environmental tests (from the same production lot) previously discussed herein, and powered at 24 VDC with specified nominal current applied in accordance with manufacturers' operating specifications. At the conclusion of the 41.6 days of steady state life testing, LED samples remained powered while the temperature chamber environment was changed from $+125^{\circ} \mathrm{C}$ down to $+24^{\mathrm{O}} \mathrm{C}$ for the purpose of collecting posttest light output data at the same temperature the initial data were collected. A visual examination was conducted in search of mechanical damage and confirmation was made that all LED samples remained operable with no notable failures found.

\section{Performance Analysis}

\section{A. Mechanical and Visual Inspection}

Upon completion of all subgroup 1 testing, LED device were mechanically inspected in accordance with dimensional requirements specified in the respective design specification using a microscope ( 10 - 60 power) with no observed failures resulting from environmental testing previously illustrated in Table 2.

\section{B. Electrical Test}

Subgroup 1 and subgroup 2 sample sets were all tested using a calibrated light meter. All LED samples were powered at 24 VDC with specified nominal current applied in accordance with manufacturers' operating specifications. Table 4 illustrates initial light output data and the end point light output data for the purpose of comparison. Likewise, Table 5 illustrates initial and the end point light output data for subgroup 2 samples resulting from steady state life testing.

Table 4. Subgroup 1 data from environmental stress testing.

\begin{tabular}{lccc} 
& Package A & Package B & Package C \\
\hline Initial Light Output Average $\left(\mu \mathrm{mol} / \mathrm{m}^{2} \mathrm{sec}\right)$ & 23.50 & 24.75 & 15.40 \\
Post Testing Light Output Average $\left(\mu \mathrm{mol} / \mathrm{m}^{2} \mathrm{sec}\right)$ & 23.60 & 24.62 & 15.43 \\
\hline Difference & -0.10 & 0.13 & -0.03 \\
Percent Change & $-0.43 \%$ & $0.54 \%$ & $-0.22 \%$
\end{tabular}

Table 5. Subgroup 2 data from steady state life test.

\begin{tabular}{lccc} 
& Package A & Package B & Package C \\
\hline Initial Light Output Average $\left(\mu \mathrm{mol} / \mathrm{m}^{2} \mathrm{sec}\right)$ & 23.82 & 24.13 & 15.71 \\
Post Testing Light Output Average $\left(\mu \mathrm{mol} / \mathrm{m}^{2} \mathrm{sec}\right)$ & 19.78 & 20.73 & 14.08 \\
\hline Difference & 4.04 & 3.41 & 1.62 \\
Percent Change & $16.97 \%$ & $14.12 \%$ & $10.34 \%$
\end{tabular}

\section{Conclusion}

Quality can be defined as conformance to specification and reliability can be defined as quality over time. O'Connor and Kleyner (2012) defined a reliability failure as termination of the ability for a product to perform the required function according to design specifications. Lenk and Lenk (2011) described two measures for reliability when conducting experimentation using LED technologies: (a) operational hours to failure, and (b) the point where an LED no longer provides sufficient light output. This particular analysis resulted in neither of these measures producing a significant failure.

Typical life studies involving electronics record number of hours until breakage or failure where this particular analysis yielded no failures for the subgroup 1 testing and yielded negligible changes in subgroup 2 steady state life samples. The percentage of light output loss observed in subgroup 2 are insignificant where Lenk and Lenk (2011) 
stressed that LED components do not necessarily fail by burning out but become dimmer over time. Therefore, an LED component should be considered an end-of-life failure when it reaches $70 \%$ of the initial light output. A review of Table 5 supported this researcher's conclusion that there were no observed failures from either subgroup 1 or subgroup 2 testing. For the purpose of selecting commercially available LED components for warm white light space applications, selected samples have provided objective evidence that these components may be selected as part of an EEE management plan with high probability that these types of technology are capable a sustaining a wide variety of harsh operating environments and are suitable for space flight and other applications requiring ruggedized design features.

Light emitting diodes are to be considered an integral part of future spacecraft design and must be evaluated for reliability as part of the environmental control and life support system (Jiank, Rodngues, Bell, Kortenkamp, \& Capristan, 2011). Jiank et al. (2011) stated that future space craft architecture must include design provisions for longer duration missions where supplies for food, water, air, and life essentials significantly increase spacecraft support costs, hence making LED technologies a viable consideration for off-setting high costs with lower volume, less energy consumption, and longer reliability.

\section{Acknowledgement}

The author would like to thank the Wisconsin Space Grant Consortium for their support of this academic partnership and recognize the University of Wisconsin -LaCrosse and physics major Paul Wedel (see Figure 11) who was instrumental in configuring the LED test vehicle discussed herein.

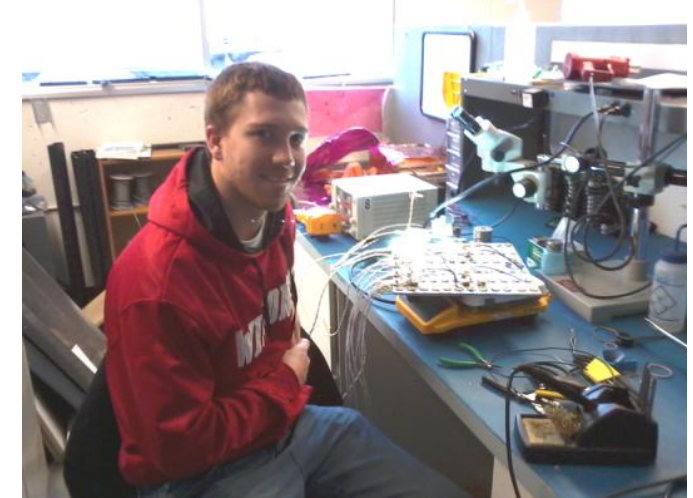

Figure 11. Paul Wedel - UW LaCrosse.

\section{References}

Brainard, G. C., Coyle, W., Ayers, M., Kemp, J., Warfied, B., Maida, J., Bowen, C., Bernecker, C., Lockley, S. W., \& Hanifin, J. P. (2012, April). Solid-state lighting for the international space station: tests of visual performance and melatonin regulation. Acta Astronautica. doi:10.1016/j.actaastro.2012.04.019

Hersman, C. \& Fowler, K. (2009, October). Best practices in spacecraft development. Mission Critical and Safety Critical Systems. 1 (3), 269-460. doi:10.1016/B978-0-7506-8567-2.00005-6

Jiank, H., Rodngues, L. F., Bell, S., Kortenkamp, D., \& Capristan, F. (2011, April). Prediction of reliability for environmental control and life support systems. Journal of Spacecraft and Rockets, 48 (2), 336 - 345. doi:10.2514/1.44792

Lenk, R., \& Lenk, C. (2011), Practical lighting design with leds. Hoboken, NJ: John Wiley and Sons, Inc.

MIL-PRF-38534 (13 September 2010). General specification for hybrid microcircuits (Revision H), FSC: 5962 United States Department of Defense, USA.

MIL-STD-883 (26 February 2010). Test method standard for microcircuits (Revision H), FSC: 5962 United States Department of Defense, USA.

Szajnfarber, A., Coles, T. M., Sondecker, G. R., Wicht, A. C., \& Weigel, A. L. (2011, August). Moon first versus flexiblepath exploration strategies: considering international contributions. Space Policy, 27 (3), 131-145. doi:10.1016/j.spacepol.2011.05.003 